\title{
Ruth Hoffmann, Stasi-Kinder - Aufwachsen im Überwachungsstaat
}

Berlin : List, 2013, 317 p., 9,99€.

Brigitte Lestrade

\section{CpenEdition}

Journals

Édition électronique

URL : http://journals.openedition.org/ifha/8388

DOI : 10.4000/ifha.8388

ISSN : 2198-8943

Éditeur

IFRA - Institut franco-allemand (sciences historiques et sociales)

Référence électronique

Brigitte Lestrade, «Ruth Hoffmann, Stasi-Kinder - Aufwachsen im Überwachungsstaat », Revue de l'IFHA

[En ligne], Date de recension, mis en ligne le 01 février 2016, consulté le 22 septembre 2020. URL :

http://journals.openedition.org/ifha/8388 ; DOI : https://doi.org/10.4000/ifha.8388

Ce document a été généré automatiquement le 22 septembre 2020.

(CIFHA 


\title{
Ruth Hoffmann, Stasi-Kinder - Aufwachsen im Überwachungsstaat
}

\author{
Berlin : List, 2013, 317 p., 9,99€.
}

Brigitte Lestrade

Depuis la chute du Mur de Berlin en 1989, beaucoup a été dit et écrit sur la défunte RDA, notamment sur la surveillance constante dont les habitants ont été l'objet de la part de la police de la sécurité de l'État, la fameuse Stasi. L'incompréhension et l'horreur, tant à l'Est qu'à l'Ouest, a été renforcée par la découverte des IM, ces «collaborateurs informels ", dont la Stasi se servait pour espionner leurs familles, leurs relations et leurs collègues de travail. L'omniprésence de ce réseau de flicage, en dépit de l'intérêt qu'il continue à susciter, n'est toutefois pas l'objet de l'ouvrage de Ruth Hoffmann. Journaliste de métier, elle s'intéressait au départ à un reportage sur les enfants ayant grandi dans un quartier huppé de Berlin, la Frankfurter Allee, là où habitaient dans les années 1970 et 1980 des représentants de la nomenklatura est-allemande. Le projet initial, la confrontation entre le monde de ces nantis avec celui des habitants estallemands lambda, s'est mué en investigation de la vie des enfants de hauts fonctionnaires, représentants et piliers de l'État. Les enfants sont adultes aujourd'hui, sont eux-mêmes parents, mais la vie qu'ils ont menée, entre privilèges et répression, les a formés, voire déformés, et placé une ombre sur le restant de leurs jours.

Ruth Hoffmann a basé son ouvrage sur une vingtaine d'interviews de descendants de membres de la Stasi, dont certains ont souhaité conserver l'anonymat. Structuré en douze chapitres qui retracent l'évolution de ces jeunes de la petite enfance au début des années 60 ou 70 jusqu'à aujourd'hui, en passant par tous les stades, de l'obéissance au doute et à la révolte, il s'accompagne, pour la plupart des chapitres, d'un complément intitulé «Exkurs » permettant de situer le vécu des enfants dans le contexte politique et social de l'époque. C'est ce qui explique le sous-titre "Grandir dans l'État de surveillance ». Rarement les rouages de la Stasi ont été analysés avec une telle connaissance détaillée du fonctionnement, un travail qui n'a été possible qu'après l'ouverture de ses archives secrètes. Ces éclairages permettent de fournir l'arrière-plan du vécu des enfants et donnent, de même, un aperçu des contraintes subies, acceptées 
ou saluées qui s'imposaient à leurs parents comme à tous les membres de la Stasi. De plus, les souvenirs des personnes interrogées sont complétés par des extraits de documents officiels, dont la RDA faisait un usage pléthorique, puisque le moindre incident, la moindre rencontre, toutes les discussions, étaient documentés, tant par les IM que par les membres de la Stasi et archivés dans les dossiers personnels de chacun. Les personnes dans le viseur de la Stasi, qu'il s'agisse de leur propre personnel et de leurs familles ou de celles considérées comme des dangers putatifs du régime, n'ont pu prendre connaissance de leur dossier qu'après la chute du Mur. Nombre d'entre eux ont appris avec effarement tout ce qui avait été collecté sur eux par des voisins, des collègues de travail, des membres de leur propre famille. C'est ce qui fait un des attraits de cet ouvrage, que d'analyser le vécu des enfants des hauts fonctionnaires de la Stasi à la lumière de leurs expériences propres et de l'écho que celles-ci ont trouvé dans les rapports rédigés sur eux à leur insu.

La surveillance dont tous les citoyens de la RDA faisaient l'objet était connue, mais on était moins au courant du fait que les membres de la Stasi étaient eux-mêmes l'objet de contrôles incessants et subissaient ce climat de méfiance et de peur qu'ils avaient créé pour l'ensemble de la population. L'originalité de cet ouvrage est de montrer comment cette atmosphère de surveillance totale, où aucune parole, aucune rencontre n'est privée, a pesé sur les enfants concernés qui, souvent, étaient laissés dans l'ignorance de la réelle activité des parents. Que pressentaient-ils? Comment réagissaient-ils au moment où, l'adolescence venue, ils découvraient la vérité ? Le quotidien de la vie de ces familles, relaté d'une plume alerte, permet au lecteur qui ne l'a pas connu, de comprendre la vie de ces enfants déchirés entre la loyauté vis-à-vis de leurs parents et le désir de s'émanciper et de prendre leurs distances. La Stasi avait demandé une implication totale et une obéissance absolue à ses membres, au travail et dans la sphère privée. Il n'est pas étonnant que peu de familles en soient sorties indemnes.

Si l'ouvrage de Ruth Hoffmann est très intéressant et informatif, il est néanmoins quelques aspects où le lecteur pourrait formuler des regrets. Ainsi, on constate que l'auteur a fait le choix de retenir pour ses interviews essentiellement des jeunes qui se sont rebellés contre le mode de vie imposé par les parents et qui en ont souffert. Il aurait certes été intéressant de voir aussi le point de vue d'enfants ayant mené une vie plus conforme aux valeurs professées par leurs parents. De plus, les parents, soumis il est vrai à des pressions énormes, sont présentés comme non seulement distants, mais souvent aussi comme violents et peu à l'écoute de leurs enfants. S'ils avaient dans l'ensemble peur pour leur carrière, qu'une parole imprudente d'un enfant aurait pu mettre en péril, on a du mal à les concevoir globalement comme de mauvais parents. Cette tendance à schématiser pourrait limiter la portée de la démonstration. Une autre remarque porte sur la structure de l'ouvrage. La méthode choisie, un va-et-vient incessant entre différentes personnes à différents stades de leur vie, interrompu de surcroît par des chapitres consacrés à l'arrière-plan politique, ne permet guère au lecteur de suivre le fil de la vie des enfants. En dépit de ces quelques observations, on peut recommander cette lecture à tout ceux qui s'intéressent à la RDA telle qu'elle fut vraiment, d'autant plus que l'auteur est parvenu à rendre la lecture agréable et non dépourvue d'un certain suspens.

Vous trouverez la table des matières ici : http://d-nb.info/1028265174/04. 
INDEX

Thèmes : Histoire des États et des pouvoirs, Histoire sociale, Histoire des mentalités Index chronologique : Époque contemporaine.

\section{AUTEUR}

\section{BRIGITTE LESTRADE}

Université de Cergy-Pontoise 